\title{
Klimaschwankungen im Pliozän von Wallensen (Hils)
}

\author{
Von P. A. Altehenger, St. Augustin bei Siegburg
}

Mit 2 Abbildungen im Text

$\mathrm{Z}$ u s a m m e $\mathrm{f}$ a s s u n g. Die pliozäne Braunkohle von Wallensen im Hils (Nordwestdeutschland) ist mit über $70 \mathrm{~m}$ Mächtigkeit im Tagebau aufgeschlossen. Der Abschnitt des Pliozäns, der als Bildungszeit der Kohle erfaßt werden kann, dürfte mehrere Jahrhunderttausende betragen.

Für die Frequenzkurven klimatisch empfindlicher Vertreter der Wallenser Pliozänflora ergeben sich bei pollenanalytischer Untersuchung der Kohle Jangfristige Schwankungen. Dadurch werden klimatische Anderungen während der Bildungszeit der Kohle sichtbar, die zwar nicht in ihrer Intensität, wohl aber in ihrem zeitlichen Verlauf mit den pleistozänen Klimaschwankungen des Postgünzglazials vergleichbar sind.

$\mathrm{S} u \mathrm{~m} m$ a ry. Exposed in the open-work, the pliocene lignite of Wallensen (Hils, Northwest Germany) can be studied by pollen analysis on a thickness of more than 70 metres. The Pliocene epoch which in this way can be explored, may amount to some hundred thousand years.

The curves of pollen frequency show long lasting oscillations owing to climatic changes during the time of formation of the lignite and suggest that these pliocene oscillations, according to their temporal rhythm, can be compared whith those of the Pleistocene, although the latter ones, at least since the Günz Glaciation, were, as we know, by far of stronger intensity than those of the Pliocene.

\section{Die Kohle von Wallensen}

Das kleine Braunkohlenlager von Wallensen liegt ungefähr in der Mitte des nordwestlichen Teiles der Hilsmulde, die hier auch geographisch als Mulde ausgebildet ist und südwestlich vom Ith, nordöstlich vom Höhenzug Thüster Berg-Duinger Berg und südöstlich vom Sandsteinmassiv des Hilsgebirges begrenzt wird. Starke Unregelmäßigkeiten in der Ausbildung der Kohlenflöze, wie auffallend differierende Fallwinkel- und Mächtigkeitsangaben, die bisher von verschiedenen Aufschlüssen vorliegen, weisen auf sehr ungleichmäßiges Absinken des Untergrundes hin. Diese Tatsache läßt zusammen mit der engen Begrenzung des Vorkommens an Salztektonik denken, wie sie bisher wiederholt vermutet (R. Herrmann 1935, W. Evers 1955, P. W. Thomson, mündlich) und neuerdings auch nachgewiesen ist (W. Th. Fratschner 1951).

Am derzeitigen Aufschluß der Südwand (1952-1956), der dieser Untersuchung zugrunde liegt, wird die Kohle durch zwei mächtige Tonmittel in ein Oberflöz und ein Unterflöz eingeteilt. Das Hangende des Oberflözes bilden quartäre Sande, das Liegende des Unterflözes tertiärer Ton; die Kohle des Mittelflözes ist stark durch Ton verunreinigt. Das Quartär liegt diskordant auf der Kohle, die mit $7^{\circ}-8^{\circ}$ nach Westen einfällt. Die Kohle selbst ist durchwegs erdig, sehr wasserhaltig (ïber 60\% durchsetzt. Das lange Zeit strittige und anderweitig nicht zu entscheidende genauere Alter konnte pollenanalytisch von P. W. Thомson $(1949,1951)$ als jüngeres Pliozän (Reuverstufe) festgelegt werden.

Für die vorliegende Untersuchung wurden aus dem ca. $76 \mathrm{~m}$ mächtigen Aufschluß 317 Schlitzproben ausgezählt, die in 20-cm-Abständen den drei Flözen entnommen sind. Die Tonmittel sind für diese Arbeit nicht mitberücksichtigt worden. Für die graphische Darstellung wurden je 10 aufeinander folgende Proben zusammengezählt und diese Summen zur besseren Vergleichbarkeit vermindert oder überhöht. Die einzelnen Werte der Kurven geben also Durchschnitte aus Horizonten von zwei Meter Mächtigkeit an.

Die Bildungszeit der Lagerstätte läßt sich nur sehr grob einschätzen. Nach Angaben verschiedener Autoren (O. Stutzer 1923, K. Pietzsch 1925, M. Schwarzbach 1950, P. W. Thomson 1951) darf als chronologisches Äquivalent für $20 \mathrm{~cm}$ Braunkohle ein Zeitraum von mindestens 600 Jahren angenommen werden, in der Regel aber wohl bedeutend 
mehr, da Stillstandslagen (im Absinken des Untergrundes), Erosionen, Waldbrände und der Inkohlungsprozeß selber den Zuwachs der Kohle sicher bedeutend verzögert haben.

Man wird also die Bildungszeit der hier untersuchten Kohle auf rund eine viertel bis eine halbe Million Jahre schätzen dürfen und kommt damit zu einem Zeitraum, der größenordnungsmäßig mit dem ganzen nachgünzeiszeitlichen Pleistozän vergleichbar ist.

\section{Die Flora}

Die pollenanalytisch faßbare Flora von Wallensen ist auffallend reichhaltig. Es lassen sich 50 Gattungen nachweisen (davon 34 mit hinreichender Sicherheit, 16 mit großer Wahrscheinlichkeit), ferner noch weitere 14 Familien (8 sicher, 6 als sehr wahrscheinlich), außerdem weitere 3 Ordnungen (davon wenigstens 2 mit Sicherheit).

Das Gesamtbild der Flora entspricht, wie schon erwähnt, der bekannten Reuver-Flora (Cl. \& E. M. REID 1915) des jüngeren Pliozäns und deckt sich gut mit den Pollenfloren von Willershausen (A. Straus 1930, 1952, 1954), Weilerswist (H. Grebe 1955) und der Wetterau (F. Kirchieimer 1934, G. Leschiк 1956). Die Floren von Willershausen und der Wetterau sind neuerdings durch Mastodon arvernensis CвоIz. \& Jов. biostratigraphisch als Jungpliozän bestätigt.

Für die vorliegende Untersuchung interessieren besonders die als Klima-Indikatoren brauchbaren Massenpollen der Waldbäume mit verschiedenen Klima-Ansprüchen. Sie lassen sich nach der Dauer ihres Verbleibens im mitteleuropäischen Raum in drei größere Gruppen einteilen: die Reuver-, die Tegelen- und die jungquartären Elemente.

$\mathrm{Zu}$ den Reuver-Elementen, die für unseren Raum mit dem kalten Prätegelen verschwinden, zählen: cf. Sequoia (= Sequoia, Cryptomeria und evtl. Metasequoia), cf. Taxodium (= Taxodium und evtl. Glyptostrobus), Sciadopitys, Liquidambar und Nyssa, wohl auch noch Cedrus und Zelkova (die in Südeuropa noch im Tegelen vorkommt).

Die Tegelen-Elemente, die bei uns die Günz-Eiszeit nicht überleben, umfassen: Tsuga, die Pinus haploxylon-Gruppe, Phellodendron, Carya, Pterocarya und möglicherweise noch Juglans und Castanea (die aber beide nur mit Vorbehalt hier genannt werden können).

$\mathrm{Zu}$ den jungquartären Elementen gehören die hier bis heute überlebenden Waldbäume: Picea, Abies, die Pinus silvestris-Gruppe; Betula, Corylus, Alnus, Carpinus, Quercus, Fagus, Salix, Ulmus, Tilit, Ilex.

Aus der übrigen Flora interessieren in diesem Zusammenhang nur noch die EricalesTetraden und die Sphagnum-Sporen, deren reichliches gemeinsames Vorkommen (in Kombinationen mit mehr oder minder reichlichem Pinuspollen) auf hochmoorartige Vegetationen hinweist. Diese sprechen für ein relativ feuchteres und kühleres Klima und sind im Wallenser Pliozän auffallend stark in der oberen Hälfte des Unterflözes vertreten.

\section{Das Klima}

\section{a) Methodik der Darstellung}

Zur Diagnose der klimatischen Verhältnisse lassen sich günstigerweise zunächst einige charakteristische Vertreter der obengenannten Gruppen in ihren quantitativen Verhältnissen relativ zueinander untersuchen.

Viele Vertreter sind hierfür ungeeignet, weil sie entweder zu selten vorkommen (also nicht „prozentbildend" sind), wie Liquidambar und Phellodendron, oder weil sie zu stark von Bodenverhältnissen abhängig sind, wie Nyssa und Taxodium, oder schließlich, weil ihre Gruppenzugehörigkeit (und damit Klima-Empfindlichkeit) nicht genügend gesichert ist, wie bei Juglans und Castanea. 


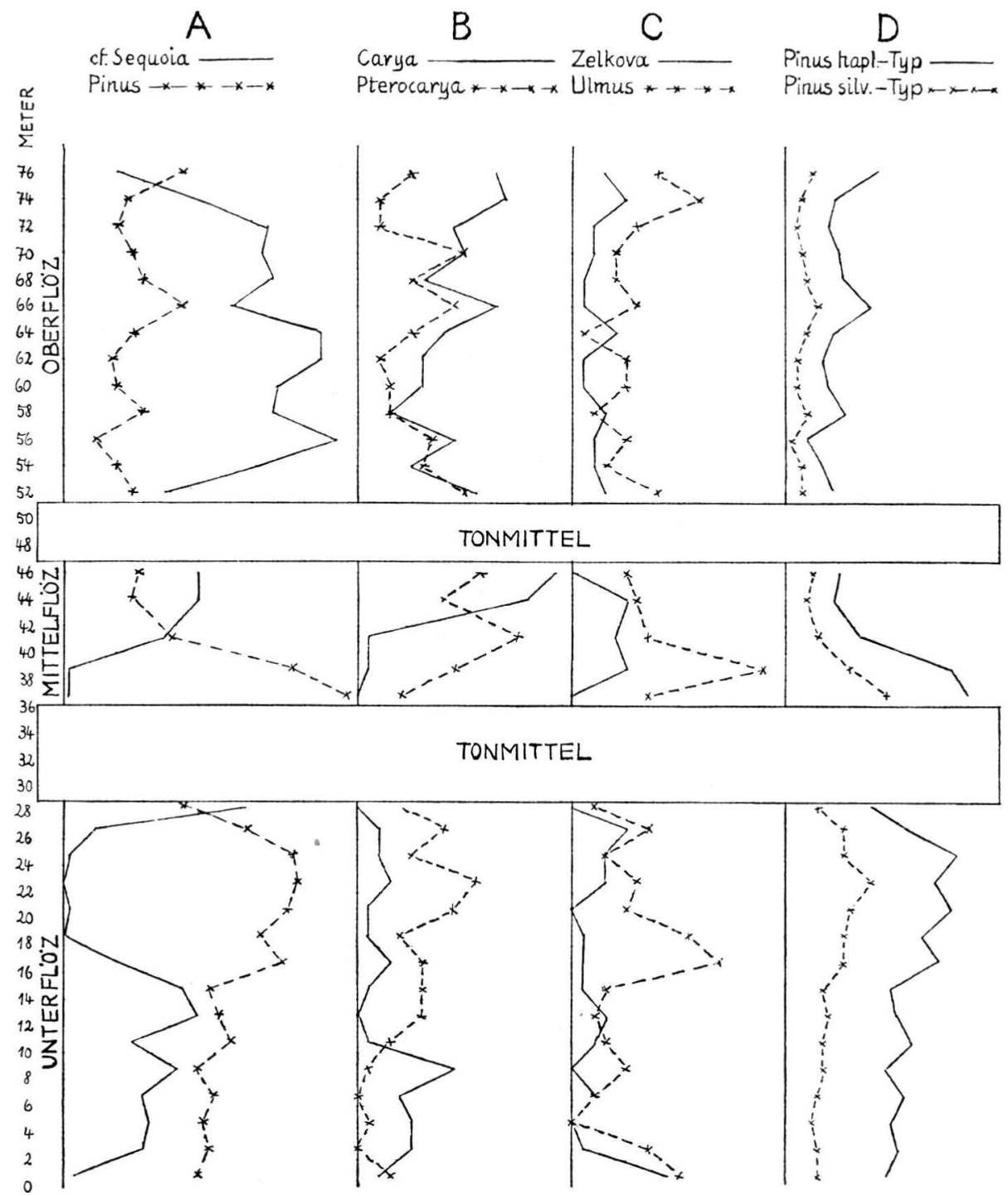

Abb. 1. Vergleich der Pollen-Frequenzkurven: A der häufigsten Koniferen, B der wichtigsten Juglandaceen, C der Ulmaceen, D der beiden Pinus-Gruppen. Die ausgezogenen Linien geben die klimatisch anspruchsvolleren Formen wieder.

Als brauchbare Indikatoren erweisen sich die im Diagramm I (Abb. 1) dargestellten Pollenstreuer: zunächst die beiden Hauptvertreter des lckalen trockneren Bruchwaldes, die tertiäre cf. Sequoia und die quartäre Pinus (=Summe aus Pinus silv.- und Pinus hapl.Gruppe), ferner die Juglandaceen (außer der sehr seltenen Jug!ans). die sonst im Miozän häufigere Carya und die für das Pliozän charakteristischere Pterocarya; ebenso die beiden Ulmaceen: die tertiäre Zelkova und die quartäre Ulmus. Die Frequenzkurven der heiden Pinusgruppen, die in Diagr. I (D) dargestellt sind, verlaufen im ganzen Aufschluß fast parallel. Eine genauere Untersuchung der Prozentverhältnisse zeigt aber auch hier einen weniger auffälligen Wechsel der Frequenz zugunsten der einen oder anderen Pinusgruppe. 
A

Reuver-Elemente:

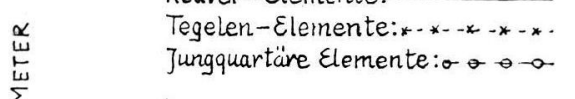

B

Summe cus Reuver-

und Tegelen-Elementen: $* x_{*}+\ldots$

Jungquartäre Elemente: $0-0-0 \rightarrow \rightarrow \rightarrow \rightarrow \rightarrow-$
76

74

72

70

68

66

64

62

60

58

56

54

52

50

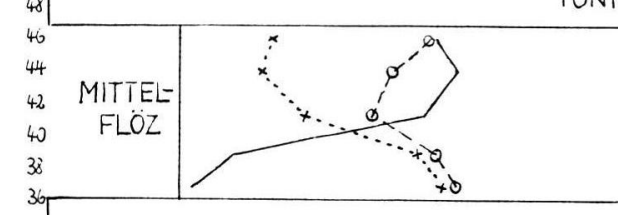

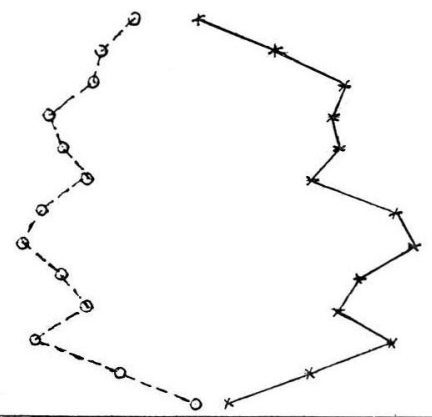

ONMITTEL

TONMITTEL

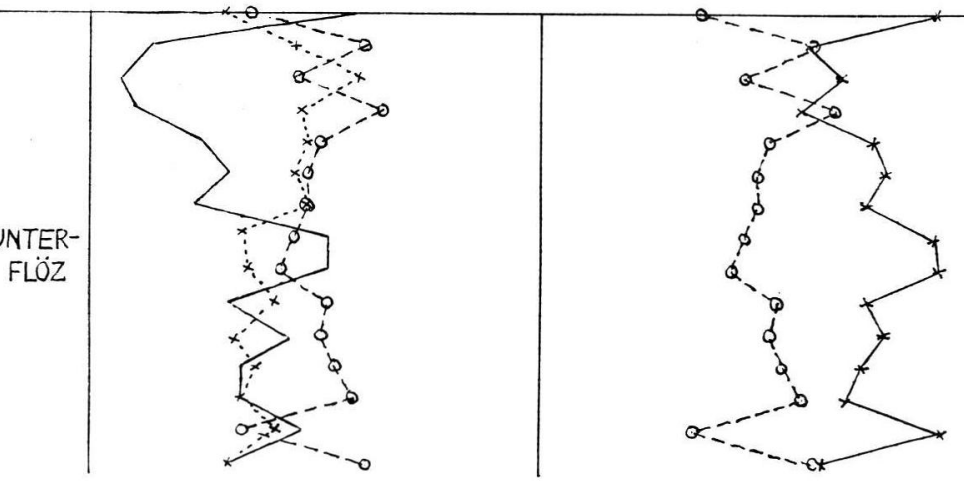

Abb. 2. Vergleich summarischer Frequenzkurven von Pollengruppen, aufgeteilt nach ihrem Verschwinden aus dem mitteleuropäischen Raum: A Reuver-Elemente, die mit dem kalten Prätegelen verschwinden, Tegelen-Elemente, die das Günz-Glazial nicht überleben, und jungquartäre Elemente, die bis heute überdauern; B Summe aus Reuver- und Tegelen-Elementen mit deutlich symmetrischem Verhalten ihres Frequenzwechsels zu den jungquartären Elementen.

Der deutlicheren Darstellung wegen wurden die Summenwerte für cf. Sequoia und Pinus durch 10 dividiert, die der Juglandaceen und Ulmaceen zweifach überhöht.

Für die Frequenzkurven des Diagramms II (Abb. 2) wurden die Summen aus sämtlichen Reuver-, Tegelen- und jungquartären Elementen verwendet einschließlich der stark von den Bodenverhältnissen (also vom Grundwasserstand) abhängigen Vertreter, wie Nyssa, Alnus u. a. Trotz der hierdurch eintretenden Verzeichnung der Klima-Abhängigkeit ergeben sich gut alternierende Kurven, deren Aussage mit dem Ergebnis des Diagramms I hinreichend übereinstimmt. 
b) Der Klimawechselin Wallensen

Nach beiden Diagrammen gliedert sich das Unterflöz deutlich in zwei Teile. Im unteren Teil, der ca. $16 \mathrm{~m}$ Mächtigkeit zeigt, sind sowohl die tertiäre cf. Sequoia wie die quartäre Pinus gut vertreten (IA); die mehr „miozäne“ Carya dominiert größtenteils über die mehr "pliozäne" Pterocarya (IB), die Kurven der (selteneren!) tertiären Zelkova und der (häufigeren!) quartären Ulmus sind verflochten (IC), ebenso die Summenkurven der Reuver-, der Tegelen- und der jungquartären Elemente (IIA). Die klimatisch anspruchsvollen Bäume sind also gut an der Gesamtsumme beteiligt, die jungquartären Elemente bleiben hinter der Summe der Reuver- und Tegelen-Elemente stets zurück (IIB). W i r dürfen danach für die Bildungszeit des unteren Unterflözes a uf ein günstiges, warmes Klima schließen.

Im oberen Unterflöz tritt eine starke Veränderung ein. Pinus dominiert jetzt völlig, cf. Sequoia tritt ganz zurïck, ebenso Carya, die von Pterocarya auffallend übertroffen wird (IA, B). Ulmus ist gegenüber Zelkova stark bevorzugt; bei Pinus tritt eine Verschiebung zugunsten der silvestris-Gruppe ein (IC, D). Die Summe der Reuver-Elemente läßt sehr nach, während die Tegelen-Elemente noch gut am Gesamtbild beteiligt bleiben (IIA). Hier finden sich auch die einzigen Überschneidungen in den Summenkurven der vor- und nachgünzeiszeitlichen Elemente (IIB). Dazu treten, wie schon erwähnt, in diesem Flözabschnitt starke Hochmoorbildungen auf. A us alledem is t a uf eine nicht gerade starke, aber doch deutliche Klimaverschlechterung zu schließen, die kïhlere und sicher auch feuchtere Verhältnisse mit sich brachte.

Die Bildungszeit des Mittelflözes erscheint in klimatischer Hinsicht wie ein Spiegelbild zu der des Unterflözes. Pinus, cf. Sequoia, die Juglandaceen und die Ulmaceen (IA, B, C) sprechen in ihrem deutlich parallelen Verhalten $f \ddot{i} r$ eine $k$ ühlere untere und eine wärmere obere Bildungsphase des Mittelflözes. Die Summenkurven der Reuver-, Tegelen- und jungquartären Elemente zeigen ein entsprechendes Bild (IIA, B).

Das Oberflöz macht demgegenüber einen eintönigeren Eindruck, der stark für ein durchgehend warmes Klima spricht. Pinus erreicht hier die niedrigsten, cf. Sequoia die höchsten Werte des ganzen Aufschlusses (IA). Besonders charakteristisch ist die weitgehende Verflechtung der Juglandaceen- und Ulmaceen-Kurven (IB, C). Das Verhältnis der Pinusgruppen ist einigermaßen deutlich zugunsten der haploxylon-Gruppe verschoben (ID). Die Reuver-Elemente dominieren weitaus stärker als sonst im Profil (IIA); die Kurven der vor- und der nachgünzeiszeitlichen Elemente divergieren hier am stärksten (IIB). Gegenüber den kühleren Bildungsphasen der beiden anderen Flöze muß während der Bildung des Oberflözes eine beträchtliche und langandauernde Klimaverbesserung ein getreten sein.

Im obersten Teil des Oberflözes ist die Aussage der Kurven nicht mehr einheitlich, da sie teils auf eine Abkühlung (IA; IIA, B), teils auf eine starke Erwärmung (IB) hinzuweisen scheinen. Vermutlich ist diese oberste Kohle durch das diskordant unmittelbar darüberliegende Quartär stark gestört.

Zusammenfassend läßt sich vom Klima des Wallenser Pliozän sagen, daß es wohl durchschnittlich etwas wärmer war, im übrigen aber ähnlich unserm heutigen gewesen sein mag. Durch das relativ reichliche Vorkommen klimatisch anspruchsvollerer Pollenstreuer im unteren Unterflöz, im oberen Mittelflöz und besonders auffallend im ganzen Oberflöz werden aber langfristige $\mathrm{klimatische} \mathrm{Schwankungen}$ sichtbar, die zwar nicht aus dem Rahmen des im ganzen warm-gemäßigten Klimas herausfal- 
len, in ihrer zeitlichen Größenordnung aber doch zu einem Vergleich mit den pleistozänen Schwankungen auffordern, da die Bildungszeit der Kohle von Wallensen, wie schon gesagt, dem nachgünzeiszeitlichen Pleistozän an Dauer vergleichbar sein dürfte.

\section{Literaturverzeichnis}

Evers, W. (1935): Zur Oberflächengestaltung des niedersächsischen Berg- und Hügellandes. 26. Jber. nieders. geol. Ver. Hannover 1935.

Fratschner, W. Th. (1951): Fazies und Tektonik im Hils. - Dissert. Clausthal 1951.

Grebe, H. (1955): Die Mikro- und Megaflora der pliozänen Ton- und Tongyttjalinse in den Kieseloolithschichten vom Swisterberg/Weilerswist (Blatt Sechtem) und die Altersstellung der Ablagerung im Tertiär der Niederrheinischen Bucht. - Geol. Jb. 70, Hannover 1955.

Herrmann, R. (1935): Das Eozän und das marine Oligozän in der Hilsmulde. - 26. Jber. nieders. geol. Ver. Hannover 1935.

Kirchheimer, F. (1934): Das Hauptbraunkohlenlager der Wetterau. - Wetterauer Gesellschaft, Hanau 1934.

Koenen, A. v. (1911): Erläuterungen zur geologischen Karte von Preußen, Lieferung 153: Blatt Salzhemmendorf. - Berlin 1911.

Leschik, G. (1956): Entstehung der Braunkohle der Wetterau und ihre Mikro- und Makroflora. Palaeontogr. B. 100, 1956.

Pietzsch, K. (1925): Die Braunkohlen Deutschlands. - Berlin 1925.

Reid, Cl. \& E. M. (1915): The Pliocene floras of the Dutch-Prussian border. - Meded. Rijksopsp. Delfst. 6, 1915.

Schwarzbach, M. (1950): Das Klima der Vorzeit. - Stuttgart 1950.

Straus, A. (1930): Dikotyle Pflanzenreste aus dem Oberpliozän von Willershausen, Krs. Osterrode, Harz. - Jb. preuß. geol. L.-A. 1930 - - (1952): Thallophythen, Kryptogamen und Gymnospermen aus dem Pliozän von Willershausen. - Ber. dtsch. bot. Ges. 65, 1952. - (1954): Beiträge zur pliozänen Flora von Willershausen IV: Die Monokotyledonen. Palaeontogr. B, 96, 1954.

Stutzer, O. (1923): Die wichtigsten Lagerstätten der Nichterze: Kohle. - Berlin 1923.

Thomson, P. W. (1949): Beitrag zur Mikropaläontologie und Waldgeschichte des Neogens (Jungtertiärs) von Niedersachsen und Schleswig-Holstein. - N. Jb. f. Min. etc., Mh., Jhrgg. 1945-1948, 1949. - - (1951): Das Interglazial von Wallensen im Hils. Eiszeitalter und Gegenwart 1, 1951.

Manuskr. eingeg. 16. 4. 1958.

Anschrift des Verf.: P. Dr. Alfons Altehenger, St. Augustin bei Siegburg (Seminar). 